\title{
Treatment Outcome of Pediatric B-ALL with Bone Marrow and Extramedullary Relapse by Anti-CD19 CAR-T
}

\author{
Xinyu Wan ${ }^{1}$, Fan Yang ${ }^{2}$, Xiaomin Yang ${ }^{3}$, tianyi Wang ${ }^{1}$, Lixia Ding ${ }^{4}$, Yan Miao ${ }^{1}$, Xiang \\ Wang $^{4}$, Yani $\mathrm{Ma}^{4}$, Chengjuan $\mathrm{Luo}^{1}$, Jingyan Tang ${ }^{5}$, Longjun $\mathrm{Gu}^{1}$, Jing Chen ${ }^{6}$, Yanjing \\ Tang $^{3}$, Jun Lu ${ }^{7}$, and Benshang $\mathrm{Li}^{1}$ \\ ${ }^{1}$ Shanghai Childrens Medical Center Affiliated to Shanghai Jiaotong University School of \\ Medicine \\ ${ }^{2}$ Department of Hematology/Oncology, Children's Hospital of Soochow University \\ ${ }^{3}$ Shanghai Children's Medical Center, Shanghai Jiao Tong University, School of Medicine \\ ${ }^{4}$ Shanghai Children's Medical Center, Shanghai Jiaotong University School of Medicine \\ ${ }^{5}$ Shanghai children's medical center \\ ${ }^{6}$ Shanghai Children's Medical Center, Shanghai Jiao Tong University School of Medicine \\ ${ }^{7}$ Children's Hospital of Soochow University
}

February 24, 2021

\begin{abstract}
Background: Anti-CD19 Chimeric Antigen Receptor T-Cell Immunotherapy (19CAR-T) has achieved impressive clinical achievements in both adult and pediatric relapsed/refractory (r/r) B-lineage acute lymphoblastic leukemia (B-ALL). However, the application and effect of CAR-T therapy in B-ALL patients with extramedullary relapse are rarely issued even disqualified in some clinical trials. Here, we examined the efficacy of 19CAR-T in patients with both bone marrow and extramedullary involvement. Methods: CAR-T cells were generated by a lentiviral vector transfection into primary human $\mathrm{T}$ lymphocytes to express anti-CD19 and anti-CD22 single chain antibody fragments (scFvs) with the cytoplasmic domains of 4-1BB and

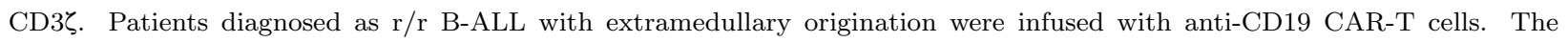
clinical responses were evaluated by bone marrow aspiration, imaging, and flow cytometry examination. Results: A total of 8 patients received 19CAR-T infusion and all of them acquired complete remission (CR), in which only 1 patient was bridged to hematopoietic stem cell transplantation (HSCT). Even though there were 3 patients relapsed after infusion, they received 19/22CAR-T infusion sequentially and acquired the second remission. To date, 5 patients are continuous CR, and all patients are still alive. The mean follow-up time was 21.9 months while the 24 -month estimated event-free survival (EFS) is $51.4 \%$. Conclusions: Anti-CD19 CAR-T therapy can lead to clinical remission for extramedullary relapsed pediatric B-ALL patients. However, the problem of CD19+ relapses after CAR-T remained to be solved. For patients relapsing after CAR-T, the second CAR-T therapy suggests creating another opportunity of remission for subsequent HSCT.
\end{abstract}

\section{Introduction}

Acute lymphoblastic leukaemia (ALL) is a leading haematological malignancy diagnosed in children all over the world. Although the long-term survival rate for paediatric ALL is approaching and even over $90 \%$, there are still $10^{\sim} 15 \%$ relapsed ALL considered as one of the leading causes of cancer mortality in children ${ }^{1,2}$. Although the relapses are mainly detected in bone marrow, it occurs occasionally that extramedullary tissues are involved accounting for 15-20\% of all the relapses ${ }^{3}$. To patients with extramedullary relapsed, the central nervous system leukemia and testicular leukaemia are the most identified ${ }^{4,5}$. However, the natural history of extramedullary relapse generally correlates with poor prognosis and limited overall survival ${ }^{6}$. 
Conventional chemotherapy, radiation, or even allogeneic hematopoietic stem cell transplantation (alloHSCT) have presented less successful in dealing with these patients, especially with multiple relapses ${ }^{7,8}$. Therefore, ALL patient with extramedullary diseases remains a great challenge and new therapeutic strategies are urgently required.

Recently, chimeric antigen receptor T-cell immunotherapy (CAR-T) is getting a wide attention and becoming an increasingly hot spot on the treatment of r/r B-ALL. CAR-T cells were first identified by Dr. Zelig Eshhar in the late 1980s and early $1990 \mathrm{~s}^{9}$. It refers to harnessing the cytotoxicity ability of genetically modified $\mathrm{T}$ cells with high specificity and cytotoxicity to tumour cells. CAR-T cells contain an antigen-specific singlechain antibody fragment $(\mathrm{scFv})$ linked to intracellular signalling domains independent of $\mathrm{MHC}$ restriction and antigen processing ${ }^{10}$. There is no doubt that 19CAR-T has achieved impressive clinical achievements in both adult and paediatric r/r B-ALL with an amazing disease remission rate, which is emerging as a standard strategy for $\mathrm{r} / \mathrm{r}$ B-ALL patients ${ }^{11-14}$. Nevertheless, the application and effect of CAR-T in B-ALL patients with extramedullary relapses are rarely issued even disqualified in some cohorts $^{11,13}$.

Here we reported our experiences of anti-CD19 CAR-T cells on the treatment of 8 pediatric B-ALL patients with extramedullary tissue and bone marrow relapse simultaneously.

\section{Methods}

Between September 2017 and July 2020, 8 cases of CD19+ B-ALL pediatric patients relapsed with both bone marrow and extramedullary infiltrations and enrolled in this study. The study was conducted in Shanghai Children's Medical Center affiliated to Shanghai Jiao Tong University School of Medicine and Children's Hospital of Soochow University and was registered at chictr.org.cn (ChiCTR2100041852). Informed consent form was signed by the guardians of patients, which required the admission of the ethics committee of Shanghai Children's Medical Center and Children's Hospital of Soochow University.

Peripheral blood (PB) was directly drawn from patients or their donor to gain $\mathrm{T}$ cells positively selected by anti-CD3-coated beads (Invitrogen). The manufacture of CAR-T cells usually took $7 \sim 10$ days. Prior to CAR-T injection, all patients were administered with lymphodepleting chemotherapy: $500 \mathrm{mg} / \mathrm{m}^{2}$ cyclophosphamide for 2 days (day- $4,-3$ ) and $40 \mathrm{mg} / \mathrm{m}^{2}$ fludarabine for 3 days (day- 4 , -3 , and 2). At day0, patients were infused fresh CAR-T cells with the dosage of $1^{\sim} 15 \times 10^{6} / \mathrm{Kg}$. Patients with severe infection were postponed the CAR-T infusion till there was no fever for 24 hours, as they were infused with cryopreserved CAR-T cells. Patients who relapsed after the first exposure to CAR-T cells were allowed to receive a second CAR-T, and administered with $500 \mathrm{mg} / \mathrm{m}^{2}$ cyclophosphamide and $50 \mathrm{mg} / \mathrm{m}^{2}$ fludarabine per day for 3 days (day- $4,-3$, and -2 ) for lymphodepletion.

Neurologic adverse events were graded according to CTCAE 4.03, and cytokine release syndrome (CRS) was graded as per the American Society of Transplant and Cellular Therapy grading criteria ${ }^{15}$. A bone marrow aspiration was done after $7 \sim 14$ days following CAR-T infusion, and disease burden assessment was performed by morphology, flow cytometry (FCM) based MRD monitoring. A negative status for MRD from bone marrow samples was defined as less than $0.01 \%$ of abnormal cells identified by multiparameter flow cytometry performed at Key Laboratory of Hematology and Oncology Ministry of Health. Imaging of the relevant extramedullary sites was performed at 1 month, 2 months, 3 months, 6 months, 1 year, 2 years, 3years following CAR-T cell infusion. CAR-T cell persistence in peripheral blood or CSF of patients was measured by the real time qPCR and copies per ug DNA was normalized by the single-copy gene, CDKN1a.

For event-free survival (EFS), an event was defined as bridging to HSCT, relapse or death. Death was the event for analysis of overall survival (OS). Kaplan-Meier curves were created for EFS and OS analysis and compared with the log-rank test. All statistics were performed as indicated using Graph-Pad Prism 8 software for Windows, version 8.0.1. Statistically significant differences were defined at a $P$ value $<0.05$.

\section{Results}

Patient characteristics were shown in TABLE 1 . Briefly, a total of 8 extramedullary relapsed childhood B-ALL patients with the average age of 7.9 years old (range, 4.3-13.0) were enrolled in this study and 
treated at Shanghai Children's Medical Center (6 patients) and Children's Hospital of Soochow University (2 patients). There were 4 patients infiltrated with CNS, 1 with testis, 2 with subcutaneous tumor and 1 with both CNS and testis. All patients were all relapsed. The mean time follow-up time was 21.9 months (5.93 to 39.97 months). In addition, 5 of 8 patients (39.4\%) relapsed more than once in which merely patient S003 relapsed after HSCT. Furthermore, all patients received fresh CAR-T product and the average infusion dose was $9.9 \times 10^{6}$ per kilogram of body weight (range, 1.8-15.0).

All 8 patients achieved complete remission (CR), in which 5 patients are continuous CR, and no CAR-T related death happened. Patients S001 was bridged to HSCT after the first CR of CAR-T. Patient-level clinical response outcomes are exhibited in Fig. 1A . The estimated EFS of patients with 19CAR-T cells was approximately $68.57 \%$ at 12 months and decreased to $51.43 \%$ at 24 months. Patients S002, S004 and S006 were relapsed with CD19+ and CD22+ blasts after prior exposure to CAR-T cells. Therefore, they sequentially accepted combined CD19 and CD22 CAR-T cells as a second CAR-T therapy at dose of $1.5 \times 10^{7}$, $4.26 \times 10^{6}$ and $1.210^{7}$ per kilogram of body weight, respectively (TABLE 2 ). Then, all of them achieved $\mathrm{CR}$ again (CR2). To date, none of them chose bridging to HSCT after the second CAR-T.

Except patient S006, CRS was observed in all patients. 3 in 8 patients were grade $3 \sim 4$. CAR-T related encephalopathy syndrome (CRES) was observed in 6 patients $(75 \%)$ and no grade $3 \sim 4$ CRES was identified. 4 patients received the tocilizumab, in which 2 patients also received glucocorticoids simultaneously. Only patient S004 received glucocorticoids intrathecal injection alone. No patient died of severe CRS or CRES (TABLE 2 ). Interleukin 6 (IL-6) and interferon $\gamma($ IFN $\gamma$ ) concentrations are main biomarkers of CRS severity. Similar patterns were observed in both IL-6 and IFN $\gamma($ Fig. 2A ), the highest level of IL-6 was $60618.45 \mathrm{pg} / \mathrm{ml}$ detected in patient S003 (TABLE 2 ). At day 7, transient increases in levels of serum IL-6 and IFN $\gamma$ occurred in most patients during CRS after CAR-T administration. We also observed that a drop of IL-6 was always followed with a lift of lymphocytes in peripheral blood (Fig. 2B ), which was temporally in accordance with the resolution of CRS.

Different from the symptoms of testicular infiltration after chemotherapy, patients had fever and the affected testis showed progressive redness and swelling $3 \sim 4$ days after CAR-T infusion, and patient may have a little pain. Although the testicle was swollen, it feels soft by physical palpation. The swelling testis reached the most serious situation in about a week, then began to decrease and continue to soften gradually until it turned close to normal size.

Patients with CNS involvement had more severe symptoms of encephalopathy manifested as high fever, headache, frequent vomiting, drowsiness, unconsciousness, hypotension or hypertension, limb trembling, convulsions. For patient with convulsions, lumber puncture was required, and the cerebrospinal fluid (CSF) protein increased significantly, meanwhile, the IL-6 concentration in CSF was much higher than that in peripheral blood. For example, on day 6, patient S004 suffered $1272.27 \mathrm{pg} / \mathrm{ml}$ of IL-6 in CSF whereas 26.62 $\mathrm{pg} / \mathrm{ml}$ in peripheral blood. We also detected higher level of CAR gene in CSF compared with peripheral blood. In patient S005, $465.026 \times 10^{3}$ copies CAR per ug DNA was detected, which was over 10 times more than that detected in peripheral blood on day 3 (Fig. 2C ). Additionally, CAR-T cells expansion were determined by real-time qPCR and the result showed that the peak CAR-T expansion was variable in different EM relapsed patients but not observed after Day 7 (Fig. 2D ).

\section{Discussion}

None of the optimal protocol is defined for treating extramedullary leukemia. Some patients still would experience multiple relapses even if they had received chemotherapy, allo-HSCT, and/or radiation ${ }^{16,17}$. Before the off-the-shelf of CAR-T therapy, blinatumomab already received regulatory approval and was widely available to treat with relapsed or refractory B-cell precursor ALL patients in clinical practice ${ }^{18,19}$. However, the presence of extramedullary relapse is associated with a lower CR rate of patients treated with blinatumomab, which is a CD19/CD3 bispecific T-cell engager antibody (BiTE) inducing cell death of CD19+ leukemia cells by redirecting CD3 $+\mathrm{T}$ cells ${ }^{20}$. Furthermore, many patients with the failure of blinatumomab still had an expression of CD1921. Two studies have presented that CD19 CAR-T therapy can eradicate leukemic 
cells in B-ALL patients with extramedullary involvement. In a study of 7 boys with testicular relapse, 6 of them remained CR in a median of 14 months (5 to 23 months) ${ }^{22}$. The group from the Children's Hospital of Philadelphia demonstrated that 6 in 10 patients with various extramedullary sites of relapse were without recurrence of disease for 6 to 13 months after CAR-T infusion ${ }^{23}$. A clinical remission in extramedullary B-ALL patients was also noted with anti-CD19 CAR-T therapy in this study.

Nevertheless, CD19+ relapse was still the most frequently occurred in the failures of 19CAR-T treatment. A trial is conducted with bispecific anti CD19/20 CAR-T as a second therapy to treat 5 anti-CD19 CAR exposed patients with B-cell lymphoma. Poor abilities of expansion in vitro and persistence were identified in

their CAR-T cells and all patients were still under disease progression state ${ }^{24}$. However, both targeted does of CAR-T cells and $100 \%$ CR were achieved in 3 relapsed patients of our study, which indicates that retreatment with CAR-T cell infusion may offer an opportunity for B-ALL pediatric patients with extramedullary relapse to wait and look for a bridge to effective HSCT. This result also suggests that combined targeting CAR-T cells can be effective to deal with this kind of immune escape problem related to tumor cells with antigen switching.

This study reveals that CD19+ relapse remains the main problem of anti-CD19 CAR-T therapy treating patients with extramedullary relapses. We envisage a second CAR-T therapy as a means to create another surviving chance for these patients and combined targeting CD19 and CD22 CAR-T therapy could be a promising choice.

\section{Acknowledgments}

This study was supported by Shanghai Collaborative Innovation Center for Translational Medicine (No. TM201928), Shanghai Shenkang Hospital Development Center (No. 16CR2024A), and the National Natural Science Foundation of China (81670174, 81670136).

\section{Conflict of interest}

All authors have no conflict of interest to this article.

\section{References}

1 Heikamp EB, Pui C-H. Next-Generation Evaluation and Treatment of Pediatric Acute Lymphoblastic Leukemia. J Pediatr. 2018;203:14-24.e2.

2 Pui C-H, Yang JJ, Hunger SP, et al. Childhood Acute Lymphoblastic Leukemia: Progress Through Collaboration. J Clin Oncol . 2015;33(27):2938-2948.

3 Ding L-W, Sun Q-Y, Mayakonda A, et al. Mutational profiling of acute lymphoblastic leukemia with testicular relapse. J Hematol Oncol . 2017;10(1):65.

4 Kosucu P, Kul S, Gunes G, Yilmaz M, Ersoz S, Ozdemir F. Multiple relapses in extramedullary localization of acute lymphoblastic leukemia.Bratislava Med J . 2012;113(01):46-49.

5 Bhojwani D, Pui C-H. Relapsed childhood acute lymphoblastic leukaemia.Lancet Oncol . 2013;14(6):e205e217.

6 KONDOH T, KURIBAYASHI K, TANAKA M, KOBAYASHI D, YANAGIHARA N, WATANABE N. CD7 promotes extramedullary involvement of the B-cell acute lymphoblastic leukemia line Tanoue by enhancing integrin 32 -dependent cell adhesiveness. Int J Oncol . 2014;45(3):1073-1081.

7 Sun W, Malvar J, Sposto R, et al. Outcome of children with multiply relapsed B-cell acute lymphoblastic leukemia: a therapeutic advances in childhood leukemia \&amp; lymphoma study. Leukemia . 2018;32(11):2316-2325.

8 von Stackelberg A, Völzke E, Kühl J-S, et al. Outcome of children and adolescents with relapsed acute lymphoblastic leukaemia and non-response to salvage protocol therapy: A retrospective analysis of the ALLREZ BFM Study Group. Eur J Cancer . 2011;47(1):90-97. 
9 Gross G, Waks T, Eshhar Z. Expression of immunoglobulin-T-cell receptor chimeric molecules as functional receptors with antibody-type specificity. Proc Natl Acad Sci . 1989;86(24):10024-10028.

10 Srivastava S, Riddell SR. Engineering CAR-T cells: Design concepts. Trends Immunol . 2015;36(8):494-502.

11 Maude SL, Laetsch TW, Buechner J, et al. Tisagenlecleucel in Children and Young Adults with B-Cell Lymphoblastic Leukemia. N Engl J Med . 2018;378(5):439-448.

12 Gardner RA, Finney O, Annesley C, et al. Intent-to-treat leukemia remission by CD19 CAR T cells of defined formulation and dose in children and young adults. Blood . 2017;129(25):3322-3331..

13 Lee DW, Kochenderfer JN, Stetler-Stevenson M, et al. T cells expressing CD19 chimeric antigen receptors for acute lymphoblastic leukaemia in children and young adults: A phase 1 dose-escalation trial.Lancet . 2015;385(9967):517-528.

14 Curran KJ, Margossian SP, Kernan NA, et al. Toxicity and response after CD19-specific CAR T-cell therapy in pediatric/young adult relapsed/refractory B-ALL. Blood . 2019;134(26):2361-2368.

15 Lee DW, Santomasso BD, Locke FL, et al. ASTCT Consensus Grading for Cytokine Release Syndrome and Neurologic Toxicity Associated with Immune Effector Cells. Biol Blood Marrow Transplant . 2019;25(4):625638.

16 Weng J, Lai P, Qin L, et al. A novel generation 1928zT2 CAR T cells induce remission in extramedullary relapse of acute lymphoblastic leukemia. J Hematol Oncol . 2018;11(1):25.

17 Pui C-H, Evans WE. A 50-Year Journey to Cure Childhood Acute Lymphoblastic Leukemia. Semin Hematol . 2013;50(3):185-196.

18 Kantarjian H, Stein A, Gökbuget N, et al. Blinatumomab versus Chemotherapy for Advanced Acute Lymphoblastic Leukemia. N Engl J Med . 2017;376(9):836-847.

19 von Stackelberg A, Locatelli F, Zugmaier G, et al. Phase I/Phase II Study of Blinatumomab in Pediatric Patients With Relapsed/Refractory Acute Lymphoblastic Leukemia. J Clin Oncol . 2016;34(36):4381-4389.

20 Aldoss I, Song J, Stiller T, et al. Correlates of resistance and relapse during blinatumomab therapy for relapsed/refractory acute lymphoblastic leukemia. Am J Hematol . 2017;92(9):858-865.

21 Jabbour E, Düll J, Yilmaz M, et al. Outcome of patients with relapsed/refractory acute lymphoblastic leukemia after blinatumomab failure: No change in the level of CD19 expression. Am J Hematol . 2018;93(3):371-374.

22 Chen X, Wang Y, Ruan M, et al. Treatment of Testicular Relapse of B-cell Acute Lymphoblastic Leukemia With CD19-specific Chimeric Antigen Receptor T Cells. Clin Lymphoma Myeloma Leuk . 2020;20(6):366-370.

23 Talekar MK, Maude SL, Hucks GE, et al. Effect of chimeric antigen receptor-modified T (CAR-T) cells on responses in children with non-CNS extramedullary relapse of CD19+ acute lymphoblastic leukemia (ALL). $J$ Clin Oncol . 2017;35(15_suppl):10507-10507.

24 Shah NN, Johnson BD, Schneider D, et al. Bispecific anti-CD20, anti-CD19 CAR T cells for relapsed B cell malignancies: a phase 1 dose escalation and expansion trial. Nat Med . 2020;26(10):1569-1575.

Table legend

TABLE 1 Patient Characteristics. \# M: male; F: female.

TABLE 2 Clinical Responses and Treatment Outcomes.++Tocilizumab. SSCorticosteroid. PP CSF level. S004 received glucocorticoids by intrathecal injection.

Figures legend 
FIGURE 1 Clinical outcomes of anti-CD19 CAR-T cells. A, Swimmer plot $(\mathrm{n}=8)$, in which each bar represents an individual patient as designated. B, Kaplan-Meier graph of EFS in 8 patients infused with 19CAR-T cells.

FIGURE 2 Changes of patients' serum biomarkers in CRS.A, The temporal relationship of IL-6 (red line) and IFN $\gamma$ (blue line) during day0 and day11.B, The temporal relationship of IL-6 (red line) and Lymphocytes (blue line) during day0 and day8; L: Lymphocytes. C, The copies of CAR gene detected in Patient S005's PB and CSF; PB, peripheral blood. D, The persistence of circulating CAR-T identified by qPCR.

\section{Hosted file}

Table 1.pdf available at https://authorea.com/users/397930/articles/510668-treatment-outcomeof-pediatric-b-all-with-bone-marrow-and-extramedullary-relapse-by-anti-cd19-car-t

\section{Hosted file}

Table 2.pdf available at https://authorea.com/users/397930/articles/510668-treatment-outcomeof-pediatric-b-all-with-bone-marrow-and-extramedullary-relapse-by-anti-cd19-car-t

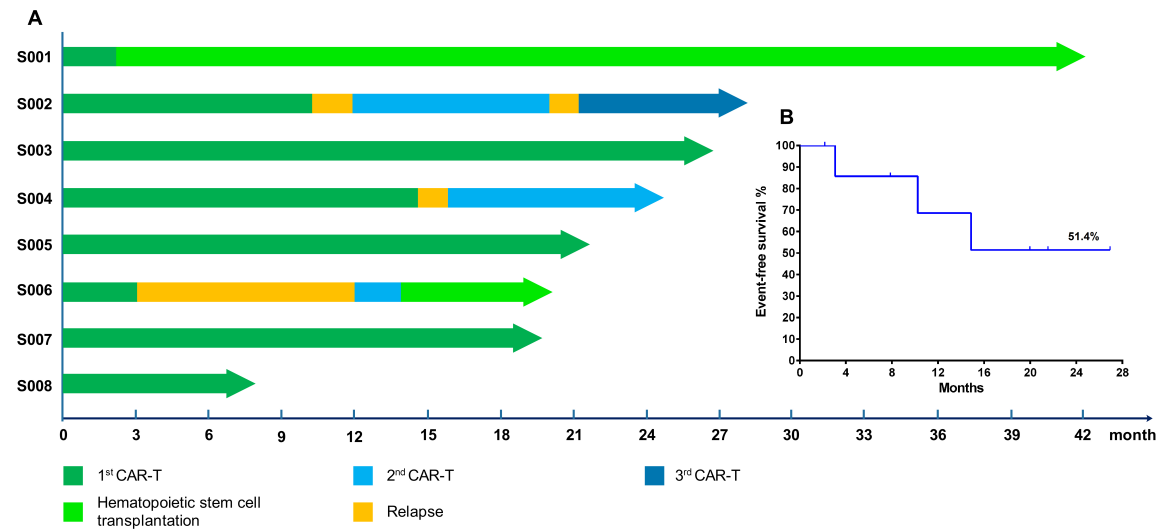


A
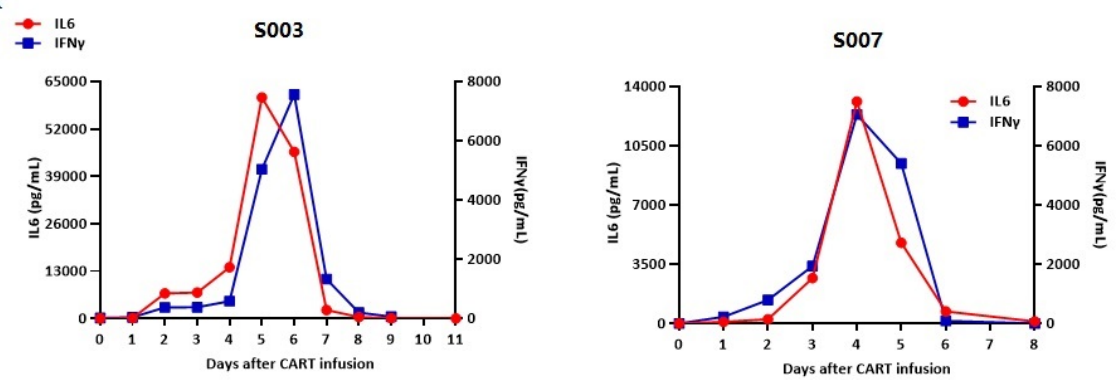

B
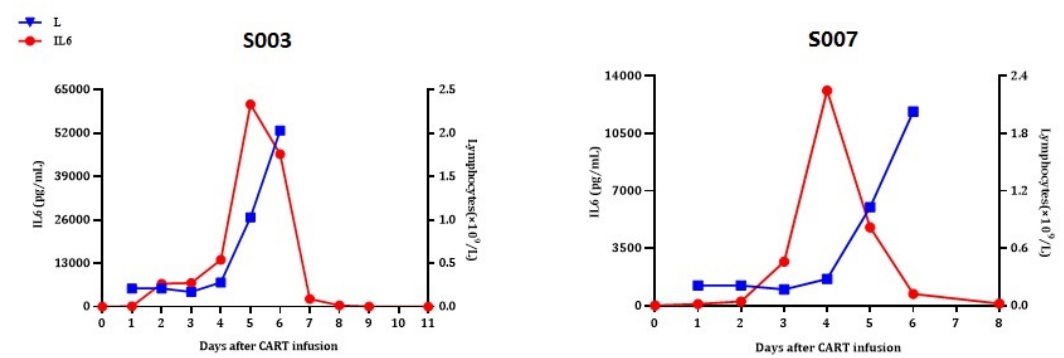

C

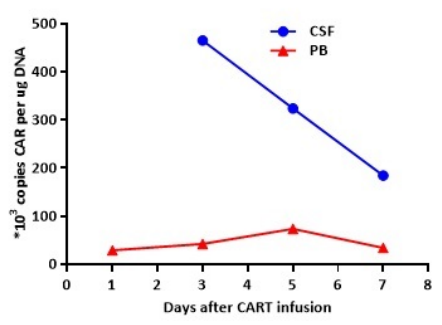

D

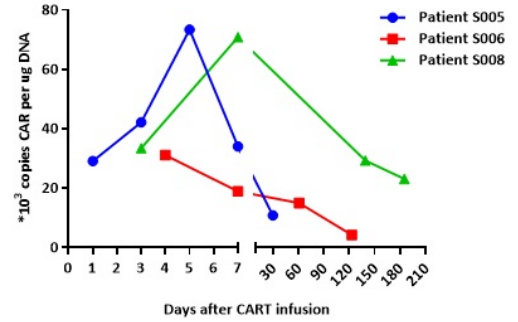

